\title{
Doença de Kikuchi-Fujimoto: apresentação de um caso
}

\section{Kikuchi-Fujimoto disease: a case report}

\author{
Jailson de Sousa Oliveira ${ }^{1}$. Gabriela Studart Galdino². \\ 1 Médico, Universidade Federal da Paraíba, Residência em Clínica Médica pelo Hospital Universitário Walter Cantídio (HUWC), \\ Universidade Federal do Ceará (UFC), Fortaleza, Ceará, Brasil. 2 Médica, Residência em Clínica Médica, Preceptora do Serviço \\ de Clínica Médica do Hospital Universitário Walter Cantídio (HUWC), Universidade Federal do Ceará (UFC), Fortaleza, Ceará, \\ Brasil.
}

\section{RESUMO}

A doença de Kikuchi-Fujimoto (DKF) ou linfadenite histiocítica necrotizante é uma doença benigna e auto-limitada, rara, caracterizada por linfadenopatia generalizada, febre e leucopenia. É um importante diagnóstico diferencial com patologias linfoproliferativas, autoimunes, infecciosas, podendo em raros casos estar associada a estas. Relatamos o caso de um paciente de 24 anos, com quadro de febre, linfadenopatia cervical e inguinal e lesões cutaneomucosas. O Fator Anti-Nuclear (FAN) foi positivo, hemograma evidenciou pancitopenia, anti-DNA negativo. A biópsia de linfonodo inguinal evidenciou linfadenite histiocítica necrotizante. O paciente evoluiu com piora clínica por atividade de doença associada a sepse, evoluindo com pericardite aguda e tamponamento cardíaco. Realizado pulsoterapia com ciclofosfamida, com melhora substancial das manifestações cutâneas e sistêmicas. O diagnóstico de pacientes com DKF deve implicar necessariamente no seguimento para avaliação do surgimento de doenças autoimunes, assim como doenças infecciosas e linfoproliferativas.

Palavras-chave: Doença de Kikuchi. Doenças raras. Linfadenite histiocítica necrosante.

\section{ABSTRACT}

The Kikuchi-Fujimoto disease (KFD) or histiocytic necrotizing lymphadenitis is a benign and self-limiting, rare disease, characterized by generalized lymphadenopathy, fever and leukopenia. It is an important differential diagnosis of lymphoproliferative disorders, autoimmune diseases, infectious diseases, and may in rare cases be associated with these. We report the case of a 24-year-old patient with fever, cervical and inguinal lymphadenopathy mucocutaneous injuries. Antinuclear antibodies were positive, blood count showed pancytopenia, negative anti-DNA. The inguinal lymph node biopsy revealed necrotizing histiocytic lymphadenitis. The patient presented clinical worsening of disease activity associated with sepsis, evolving with acute pericarditis and cardiac tamponade. Held cyclophosphamide pulse therapy, with substantial improvement of skin and systemic manifestations. The diagnosis of patients with KFD necessarily implies the follow-up for evaluation of the appearance of autoimmune diseases and infectious and lymphoproliferative disorders.

Keywords: Kikuchi Disease. Rare diseases. Histiocytic necrotizing lymphadenitis.

Autor correspondente: Jailson de Sousa Oliveira, Avenida Historiador Raimundo Girão, 630, apartamento 1506, Meireles, Fortaleza, Ceará. CEP: 60165-050.Telefone: +55 85 99656-2582. E-mail: jsomed2012@gmail.com

Conflito de interesses: Não há qualquer conflito de interesses por parte de qualquer um dos autores.

Recebido em: 10 Set 2016; Revisado em: 04 Nov 2016; Aceito em: 21 Nov 2016. 


\section{INTRODUÇÃO}

ADoençadeKikuchi-Fujimoto(DKF)oulinfadenitehistiocítica necrotizante é uma doença rara, benigna e auto-limitada, de etiologia desconhecida, que afeta principalmente mulheres jovens. Apresenta-se com linfadenopatia generalizada, predominantemente, na região cervical, acompanhada de febre e leucopenia em até $50 \%$ dos casos. ${ }^{1}$

Na Doença de Kikuchi-Fujimoto (DKF), as manifestações cutâneas são frequentes e geralmente apresentam características histopatológicas semelhantes às observadas nos linfonodos envolvidos. A presença de linfadenite histiocítica necrotizante em exame histopatológico confirma o diagnóstico de doença de Kikuchi-Fujimoto. Esta tem sido descrita em pacientes com lúpus eritematoso sistêmico (LES), e, por outro lado, pacientes saudáveis que desenvolvem DKF autolimitada podem evoluir para LES após vários meses. ${ }^{2}$

Esse estudo tem como objetivo o relato de caso de um paciente com uma doença rara, subdiagnosticada, que pode em alguns casos, ter uma morbidade considerável e associação com doenças autoimunes e infecciosas.

\section{RELATO DO CASO}

Paciente, 24 anos, natural e procedente de Trairi-CE, solteiro, ajudante de mecânico. Admitido com queixa principal de dor na boca e dificuldade para engolir há 20 dias. Após 2 a 3 dias a boca se tornou dolorosa ao toque e sangrante a manipulação. Após uma semana de evolução, paciente referiu que se associou aos sintomas uma dor de moderada intensidade em orelha direita com drenagem de uma secreção mucopurulenta, associada a diminuição da acuidade auditiva, além de episódios febris diários, sem calafrios, mensurados em $38-39^{\circ} \mathrm{C}$, que cediam com uso de antitérmico.

Há 01 ano o paciente apresentou um quadro de astenia, dispneia aos esforços, hiporexia e edema generalizado, tendo sido diagnosticado com hipotireoidismo. Relatava tabagismo por 10 anos, cerca de cinco cigarros/dia. Referia etilismo de cerca de 2 litros de destilados por fim de semana por 10 anos. Vida sexual ativa, cerca de cinco parceiras, com uso de preservativo.

No exame admissional estava hipocorado $(2+/ 4+)$, edema de face $(1+/ 4+)$, linfonodomegalia em região submandibular direita, $1 \mathrm{~cm}$ de diâmetro, fibroelático, móvel, indolor, linfonodomegalias inguinais bilaterais (maior medindo 1,5 $\mathrm{cm}$, móvel, fibroelástico, doloroso a mobilização), mucosa oral ingurgitada, aspecto friável, com lesões esbranquiçadas em palato mole, região posterior do lábio superior (Figuras 1 e 2). Diante das hipóteses de candidíase esofágica, otite média aguda e hipotireoidismo descompensado, foram prescritos antibiótico, antifúngico e levotiroxina.

Nos exames complementares iniciais, foi evidenciado anemia normocítica e normocrômica, velocidade de hemossedimentação de $120 \mathrm{~mm} / \mathrm{h}$ e creatinina: $1,4 \mathrm{mg} / \mathrm{dL}$. Durante internação, começaram a surgir lesões genitais bolhosas de conteúdo citrino, com formação de crostas posteriormente e lesões orais que evoluíram para úlceras, sendo iniciado aciclovir para tratamento de herpes zoster. Paciente foi submetido à endoscopia digestiva alta que evidenciou úlceras esofágicas sugestivas de lesões herpetiformes, no entanto a biópsia duodenal descartou doença celíaca.

Figura 1. Lesões em mucosa oral vesico-bolhosas, sangrantes, hiperemiadas, associadas a crostas.

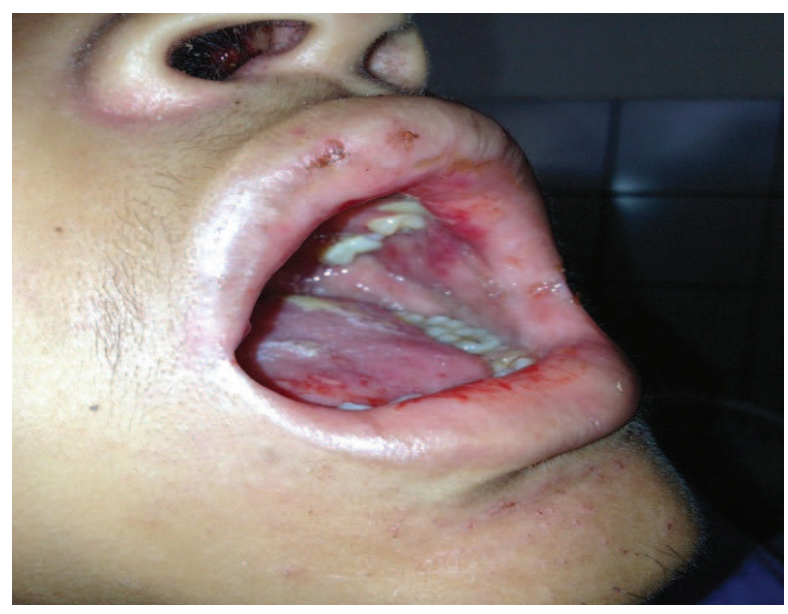

Figura 2. Língua despapilada, com lesões esbranquiçadas e lesões vesico-bolhosas.

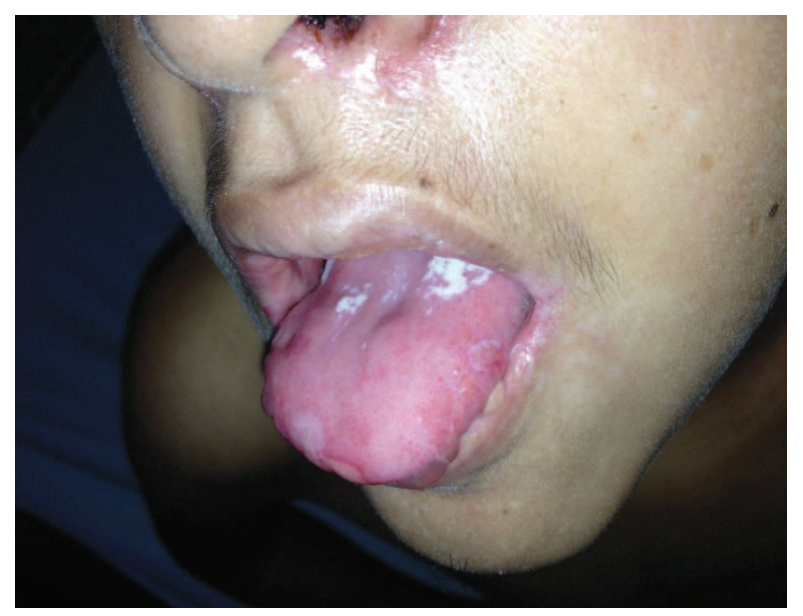

O paciente evoluiu com febre diária, hiporexia, odinofagia, piora das lesões orais, linfonodomegalias generalizadas e dor abdominal intensa. A ultrassonografia de abdome evidenciou linfonodomegalias mesentéricas e ilíacas, hepatomegalia homogênea e ascite de pequeno volume. Exames laboratoriais: hemoglobina: 9,0 g/dL; hematócrito: 27\%; leucócitos: 3250/ $\mathrm{mm}^{3}$; neutrófilos: $1120 / \mathrm{mm}^{3}$; linfócitos: $802 / \mathrm{mm}^{3}$; plaquetas:

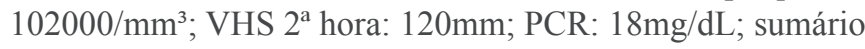
de urina: $1+$ de proteína; FAN reagente 1:1280 padrão nuclear pontilhado; anti-DNA, anti-Ro e anti-La negativos; 
anti-Sm: indeterminado; C4: $10 \mathrm{mg} / \mathrm{dL}$ (valor de referência: 19-52 mg/dL); C3: 36mg/dL (valor de referência: 87-200 mg/ $\mathrm{dL}$ ); fator reumatoide: $23 \mathrm{UI} / \mathrm{mL}$ (valor de referência $<14 \mathrm{UI} /$ $\mathrm{mL}$ ); Ferritina: 1650ng/ml; VDRL: não reagente; sorologias para HIV, HTLV I e II, hepatites B e C negativas. Foram aventadas as hipóteses de lúpus eritematoso sistêmico (LES) ou doença linfoproliferativa. Diante da piora do paciente foi decidido realizar pulsoterapia de metilprednisolona 500mg por 3 dias e iniciar antibióticos (piperacilina/tazobactam e teicoplanina) para o quadro de infecção secundária em lesões genitais e odinofagia intensa.

Após quatro dias do início da pulsoterapia o paciente apresentou piora clínica, com surgimento de lesões bolhosas disseminadas (Figura 3), febre persistente, dispneia, dor pleurítica, taquicardia e hipofonese de bulhas. Realizou ecocardiograma transtorácico que evidenciou derrame pericárdico importante, com sinais de tamponamento cardíaco, sendo realizada pericardiocentese e biópsia pericárdica. Uma biópsia do linfonodo foi compatível com linfadenite histiocítica necrotizante definindo o diagnóstico de doença de Kikuchi-Fujimoto.

Figura 3. Lesões bolhosas, algumas com evolução para crostas e despigmentação cutânea disseminada, predominantemente em tronco e membros.

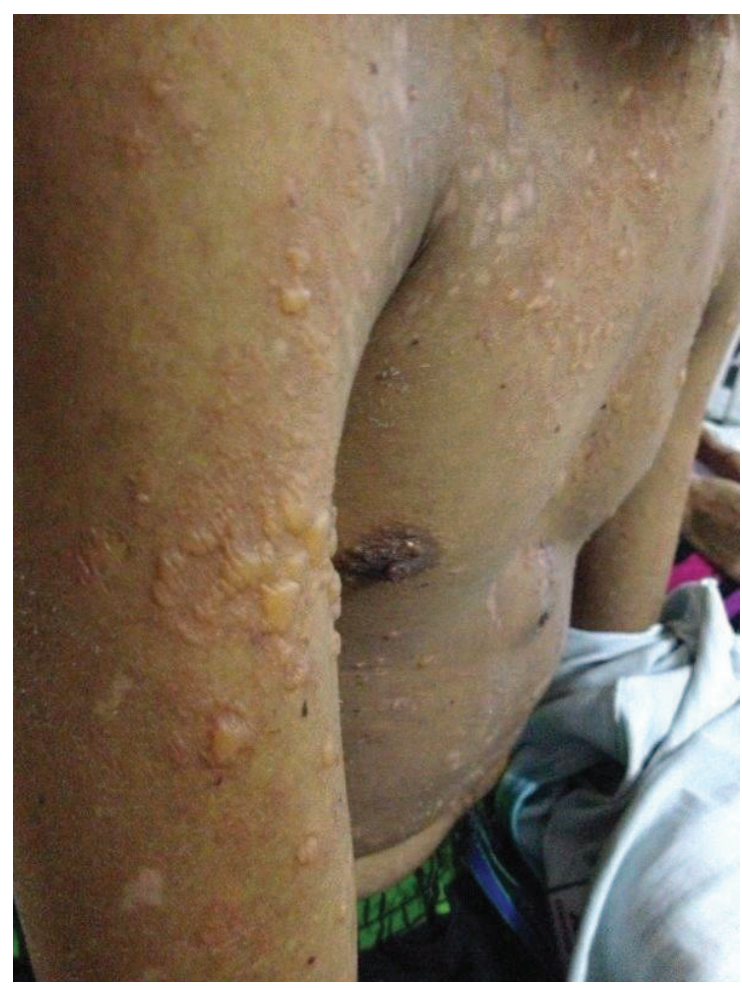

Foi completado o tratamento com piperacilina/tazobactam e teicoplanina por 14 dias e iniciado pulsoterapia com ciclofosfamida venosa $500 \mathrm{mg}$ a cada 2 semanas. O paciente evoluiu com melhora clínica importante, com resolução do quadro cutâneo e sistêmico, assim como normalização de exames laboratoriais, após 3 ciclos de ciclofosfamida. Recebeu alta hospitalar e após 6 ciclos de ciclofosfamida foi iniciado azatioprina $50 \mathrm{mg} /$ dia ambulatorialmente.

\section{DISCUSSÃO}

A doença de Kikuchi-Fujimoto se caracteriza pelo comprometimento de linfonodos cervicais (90\%), muitas vezes no contexto de poliadenopatia $(52 \%)$, associada à hepatomegalia e esplenomegalia em 14,8\% dos casos. A presença de sintomas constitucionais como febre (67\%), astenia $(74,4 \%)$, e perda de peso $(51,2 \%)$ também tem sido observada. Outras manifestações podem incluir erupção cutânea (32,9\%), artralgia $(34,1 \%)$, e mais raramente casos de meningite asséptica e síndrome hemofagocítica. As principais alterações laboratoriais incluem linfopenia $(63,8 \%)$ e aumento de proteínas de fase aguda $(56,4 \%){ }^{3}$ Tais manifestações condizem com o caso relatado, principalmente no que diz respeito aos sintomas constitucionais e manifestações cutaneomucosas.

A etiologia da doença de Kikuchi-Fujimoto ainda não é bem compreendida, mas acredita-se que possa haver uma resposta hiperimune ou autoimune desencadeada por uma infecção de diversos agentes infecciosos, tais como HIV, herpesvírus tipos 6 e 8, Epstein-Barr Vírus, Yersínia e toxoplasma. ${ }^{4}$

Na doença de Kikuchi-Fujimoto, os linfonodos das cadeias cervicais são os mais afetados. A adenopatia, às vezes, pode ser dolorosa ou sensível à palpação. Até $22 \%$ dos pacientes podem apresentar linfadenopatia generalizada, enquanto a hepatoesplenomegalia está presente apenas em pequena porcentagem. ${ }^{5}$ No caso descrito, o paciente apresentou linfadenopatia cervical, inguinal e hepatomegalia.

Deve-se ressaltar que o diagnóstico de DKF foi confirmado pela biópsia de linfonodo, demonstrando linfadenite histiocítica necrosante. A biópsia é imprescindível para que sejam excluídas outras doenças especialmente infecciosas, linfoproliferativas, autoimunes e hematofagocíticas que podem ter associação com DKF. A presença de infecções virais ou bacterianas pode deflagrar o quadro inicial da doença de Kikuchi-Fujimoto, mas sem associação causal com esta. ${ }^{6}$

A linfadenopatia de Kikuchi-Fujimoto também pode ser morfologicamente indistinguível do acometimento linfonodal no LES. Se a linfadenopatia, acompanhando o LES, apresentar extensa necrose e não houver nenhum outro dado clínico, a não ser a linfadenomegalia, será difícil a diferenciação. A confirmação clínica dos critérios de LES, como diminuição do complemento sérico, presença de anticorpos antinucleares mais específicos da doença (anti-Sm e anti DNA) e a biopsia linfonodal podem ajudar no diagnóstico diferencial. ${ }^{7}$

ADKF tem sido raramente relatada em associação com doenças autoimunes, doença mista do tecido conjuntivo, síndrome antifosfolipídeo, tireoidite, polimiosite, esclerodermia e LES. DKF foi relatada entre $4 \%$ e $13 \%$ na população com LES, ocorrendo principalmente por ocasião do diagnóstico de LES. Além disso, tem-se sugerido em outros trabalhos que DKF e LES exibam superposição das manifestações, com os mesmos achados histológicos e mecanismos autoimunes. ${ }^{8}$ 
Embora a associação entre lúpus eritematoso sistêmico e DKF seja pouco descrita, o número de relatos de DKF em pacientes com LES com acometimento cutâneo é crescente. Em pacientes que tiveram início simultâneo de ambas as doenças, lúpus bolhoso era comumente observado, sugerindo que a DKF em pacientes com LES apresenta-se com manifestações cutâneas. ${ }^{9}$

DKF é causa rara de linfadenopatia associada à febre de origem indeterminada, afetando em sua maioria mulheres jovens, com evolução benigna e autolimitada, na maioria dos casos. No caso da associação com o LES, o diagnóstico pode ser simultâneo ou uma doença ser anterior à outra. $\mathrm{O}$

\section{REFERÊNCIAS}

1. Martins SS, Buscatti IM, Freire PS, Cavalcante EG, Sallum AM, Campos LM, et al. Kikuchi-Fujimoto disease prior to childhoodsystemic lupus erythematosus diagnosis. Rev Bras Reumatol. 2014;54(5):400-3.

2. Paradela S, Lorenzo J, Martínez-Gómez W, Yebra-Pimentel T, Valbuena L, Fonseca E. Interface dermatitis in skin lesions of Kikuchi-Fujimoto's disease: a histopathological marker of evolution into systemic lupus erythematosus? Lupus. 2008;17(12):1127-35.

3. Dumas G, Prendki V, Haroche J, Amoura Z, Cacoub P, Galicier L, et al. Kikuchi-Fujimoto disease: retrospective study of 91 cases and review of the literature. Medicine (Baltimore). 2014;93(24):372-82.

4. Antunes I, Botella A, Marques F, Araújo I, Abreu A, Cardiga R, et al. Dilemas de diagnóstico de linfadenite necrotizante, Acta Med Port. 2011; 24(Supl. 3): 681-4.

5. Martínez-Vázquez C, Hughes G, Bordon J, Alonso-Alonso J, Anibarro-Garcia A, Redondo-Martínez E, et al. Histiocytic diagnóstico diferencial deve ser feito com outras causas de linfadenite, especificamente doenças infecciosas, neoplásicas e auto-imunes. Suspeita-se de mecanismos patogenéticos semelhantes na DKF e no LES; acredita-se que ocorra ativação linfocitária desencadeada por um mesmo fator causal, mas, provavelmente não existe uma causa única para a DKF. ${ }^{10}$

A Doença de Kikuchi-Fujimoto é uma doença rara, pouco conhecida e subdiagnosticada no Brasil. Pode ter um curso benigno, devendo ser considerada como diagnóstico diferencial de adenopatias, uma vez que pode estar associada a doenças autoimunes, infecciosas ou linfoproliferativas.

necrotizing lymphadenitis, Kikuchi-Fugimoto's disease, associated with systemic lupus erythemotosus. QJM. 1997;90(8):531-3.

6. Blewitt RW, Kumar SN, Abraham JS. Recurrence of Kikuchi's lymphadenitis after 12 years. J Clin Pathol. 2000;53(2):157-8.

7. Kucukardali Y, Solmazgul E, Kunter E, Oncul O, Yildirim S, Kaplan M. Kikuchi-Fujimoto disease: analysis of 244 cases. Clin Rheumatol. 2007;26(1):50-4.

8. Poulose V, Chiam P, Poh WT. Kikuchi's disease: a Singapore case series. Singapore Med J. 2005;46(5):229-32.

9. Kim SK, Kang MS, Yoon BY, Kim DY, Cho SK, Bae SC, et al. Histiocytic necrotizing lymphadenitis in the context of systemic lupus erythematosus (SLE): Is histiocytic necrotizing lymphadenitis in SLE associated with skin lesions? Lupus. 2011;20(8):809-19.

10. Ogata S, Bando Y, Saito N, Katsuoka K, Ishii M. KikuchiFujimoto disease developed into autoimmune disease: a report of two cases. Mod Rheumatol. 2010;20(3):301-5.

\section{Como citar:}

Olivieira JS, Galdino GS. Doença de Kikuchi-Fujimoto: apresentação de um caso. Rev Med UFC. 2017 mai-ago;57(2):53-56. 